\title{
Weakly dissipative systems in Celestial Mechanics
}

\author{
Alessandra Celletti ${ }^{1}$ \\ Dipartimento di Matematica, Università di Roma Tor Vergata, Via della Ricerca \\ Scientifica 1, I-00133 Roma (Italy) celletti@mat.uniroma2.it
}

\begin{abstract}
Summary. We investigate the dynamics associated to nearly-integrable dissipative systems, with particular reference to some models of Celestial Mechanics which can be described in a weakly dissipative framework. We start by studying some paradigmatic models provided by the dissipative standard maps in 2 and 4 dimensions. The dynamical investigation is performed applying frequency analysis and computing the differential fast Lyapunov indicators. After recalling a few properties of adiabatic invariants, we provide some examples of nearly-integrable dissipative systems borrowed from Celestial Mechanics, and precisely the spin-orbit coupling and the 3-body problem. We conclude with a discussion on the existence of periodic orbits in dissipative autonomous and non-autonomous systems.
\end{abstract}

\section{Introduction}

Celestial Mechanics provides a plethora of physical examples that are described by nearly-integrable dissipative dynamical systems. For instance, the celebrated three-body problem is known to be non-integrable, though in many applications it can be considered close to an integrable system; however, the conservative setting is not always sufficient to describe the dynamics: accurate investigations of the motion of the celestial objects often require to take into account dissipative effects, like the solar wind, the Yarkowsky effect or the radiation pressure. Nevertheless in many situations the dissipative effects are much less effective than the conservative contribution: for this reason we can speak of a nearly-integrable weakly dissipative three-body problem. Another example with similar features is the spin-orbit problem, concerning the motion of a rotating ellipsoidal satellite revolving on a Keplerian orbit around a central body. In this case the conservative setting is described by a nearlyintegrable problem, which is ruled by a perturbing parameter representing the equatorial oblateness of the satellite. The internal non-rigidity of the satellite provokes a tidal torque, whose effect is typically much smaller than the conservative part. 
In order to approach the analysis of the dissipative nearly-integrable systems, we start by investigating a simple discrete model known as the dissipative standard map (see [3], [4], [6], [8], [20], [29], [32]). Its dynamics is studied through frequency analysis $([21],[22])$ and by means of a quantity called the differential fast Lyapunov indicator as introduced in [8]. We remark that these approaches can be easily adapted to higher dimensional mappings as well as to continuous systems. By means of these techniques we analyze the occurrence of periodic attractors and of invariant curve attractors as the characteristic parameters of the system are varied. The results obtained for the standard map allow an easier approach to continuous systems; indeed after reviewing some results on the adiabatic invariants for a dissipative pendulum, we start by exploring some paradigms of nearly-integrable dissipative systems borrowed from Celestial Mechanics. In particular, we focus on the spin-orbit interaction for which we present some explicit expressions of dissipative forces known as MacDonald's and Darwin's torques. In this context we discuss the occurrence of capture into resonance, which depends on the specific form of the dissipation (see, e.g., [10], [12], [15], [16]).

We also provide a short discussion of the restricted planar, circular, 3-body problem and related sources of dissipation (see, e.g., [1], [2], [25], [31]). We conclude by mentioning some results about the existence of periodic orbits in (dissipative) autonomous and non-autonomous systems (compare with [27], [28], [9], [30]).

\section{The dissipative standard map}

A simple model problem which inherits many interesting features of nearlyintegrable dissipative systems is given by the so-called generalized dissipative standard map, which is described by the equations

$$
\left\{\begin{array}{l}
y^{\prime}=b y+c+\frac{\varepsilon}{2 \pi} s(2 \pi x) \\
x^{\prime}=x+y^{\prime}
\end{array}\right.
$$

where $y \in \mathbf{R}, x \in[0,1), b \in \mathbf{R}_{+}, c \in \mathbf{R}, \varepsilon \in \mathbf{R}_{+}$and $s(2 \pi x)$ is a periodic function. In the case of the classical standard map one defines $s(2 \pi x)=$ $\sin (2 \pi x)$. The quantity $\varepsilon$ is referred to as the perturbing parameter and it measures the nonlinearity of the system. The parameter $c$ is called the drift parameter and it is zero in the conservative setting. Finally, $b$ is named the dissipative parameter, since the Jacobian of the mapping is equal to $b$. Indeed, for $b=1$ one reduces to the conservative case, $0<b<1$ refers to the (strictly) dissipative case, while for $b=0$ one obtains the one-dimensional sine-circlemap given by $x^{\prime}=x+c+\varepsilon s(2 \pi x)$.

In the conservative case the dynamics is ruled by the rotation number $\omega \equiv$ $\lim _{j \rightarrow \infty} \frac{x_{j}-x_{0}}{j}$; indeed, if $\omega$ is rational the corresponding dynamics is periodic, while if $\omega$ is irrational, the corresponding trajectory describes for $\varepsilon$ sufficiently small an invariant curve on which a quasi-periodic motion takes place. 
In the dissipative setting it is useful to introduce the quantity

$$
\alpha \equiv \frac{c}{1-b}
$$

and we immediately recognize that for $\varepsilon=0$ the trajectory $\{y=\alpha\} \times T^{1}$ is invariant. Notice that $c=\alpha(1-b)=0$ for $b=1$ (i.e., in the conservative case).

In the following we shall specify the function $s(2 \pi x)$ by taking $s_{1}(2 \pi x)=$ $\sin (2 \pi x)$ or $s_{1,3}(2 \pi x)=\sin (2 \pi x)+\frac{1}{3} \sin (2 \pi x \cdot 3)$. Moreover, we shall take $\alpha$ as the golden mean, $\alpha=\frac{\sqrt{5}-1}{2}$, or as a rational number.

By iterating one of the above mappings, different kinds of attractors appear: invariant curves, strange attractors and periodic orbits, characterized by different values of the largest Lyapunov exponent. Figure 1 reports the dynamics of the mapping (1) with $s(2 \pi x)=\sin (2 \pi x)$ and $\alpha=0.2, \varepsilon=0.8$, for different values of the dissipative parameter; a transient of 10000 iterations is preliminary performed to get closer to the attractor. For $b=0.1$ one observes an invariant curve attractor, while a piecewise attractor appears for $b=0.2718$ and a periodic orbit attractor (denoted with crosses) is evident for $b=0.28$.

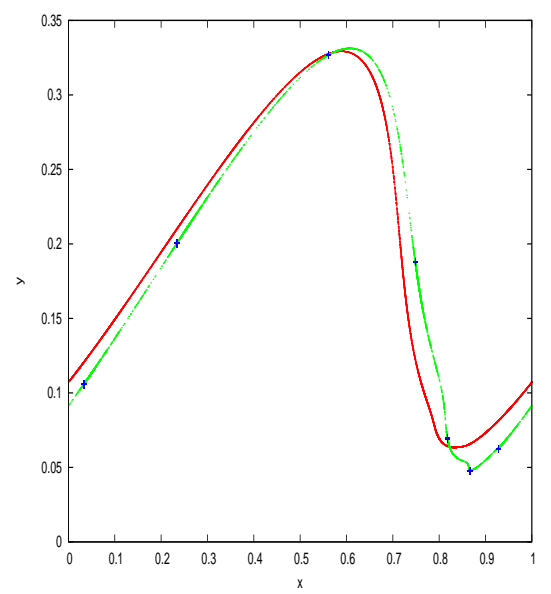

Fig. 1. Attractors of (1) with $s(2 \pi x)=\sin (2 \pi x), \alpha=0.2, \varepsilon=0.8$, while $b$ takes the values $0.1,0.2718,0.28$.

The fate of the trajectories of the dissipative mapping is rather intriguing. Indeed, orbits might wander the phase space running in zigzags through tori and chaotic separatrixes or it may happen that the motion is permanently captured into a resonance. An example is shown in Figure 2 which reports the evolution of the dynamics associated to the mapping (1) with $s(x)=s_{1}(x)$ and for $\alpha=\frac{\sqrt{5}-1}{2}$. The perturbing parameter is set to $\varepsilon=0.8$, while the dissipation is fairly weak, being $b=1-0.00001$. Taking the initial values 
$\left(y_{0}, x_{0}\right)=(5,0)$, the left panel of Figure 2 shows that the evolution of $10^{5}$ iterates escapes many resonances before being captured by the 3:2 resonance (at approximately $y_{0}=1.5$ ). Starting from the last of the previous $10^{5}$ iterates, we perform some more $5 \cdot 10^{5}$ iterations (see the right panel of Figure 2) which manifest a spiralling toward the equilibrium point, after jumping across some secondary resonances. Further iterations would lead to end-up on the center of the resonance.
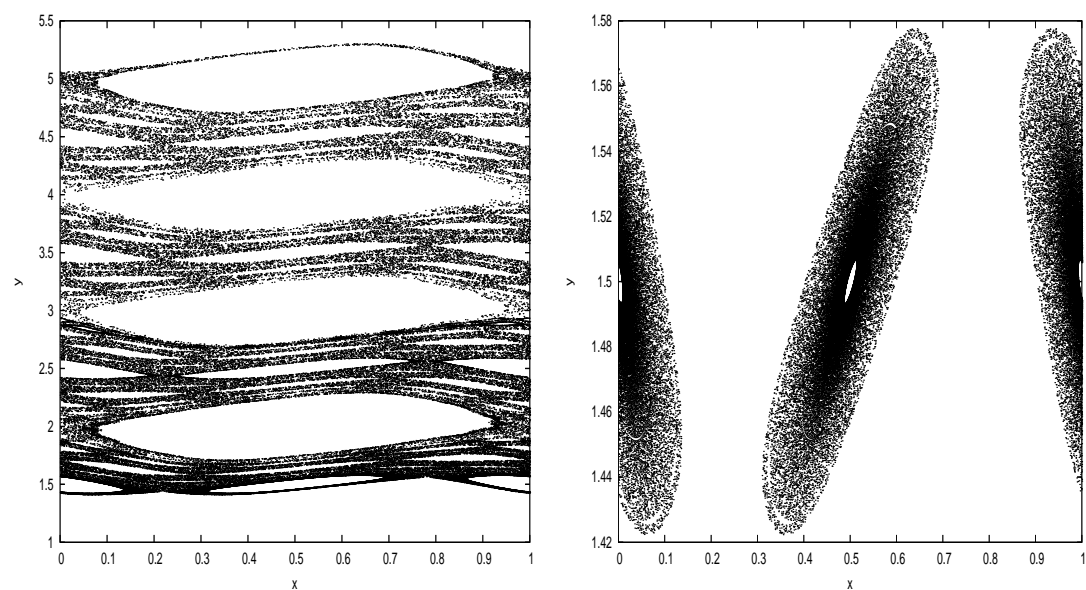

Fig. 2. Capture into resonance for the mapping (1) with $s(2 \pi x)=s_{1}(2 \pi x), \alpha=$ $\frac{\sqrt{5}-1}{2}, \varepsilon=0.8, b=1-0.00001,\left(y_{0}, x_{0}\right)=(5,0)$. Left panel: the first $10^{5}$ iterates; right panel: some more $5 \cdot 10^{5}$ iterations.

\section{Techniques for the numerical investigation of the dynamics}

In order to analyze the dynamics of the dissipative standard map we implement two complementary numerical techniques, which are based on the frequency analysis (see, e.g., [21], [22], [23]) and on the computation of the so-called fast Lyapunov indicators (see, e.g., [13], [14], [17]), modified in order to work in the dissipative case. We refer the reader to [8] for a complete description and implementation of these techniques.

\subsection{Frequency analysis}

Frequency analysis relies on the computation of the frequency of motion, which is determined by applying the following algorithm. For a given conservative $2-$ dimensional mapping $M$, let us denote by $P_{n}=M^{n}\left(P_{0}\right)$ the $n$-th iterate of the 
point $P_{0}$ which we assume to belong to an invariant curve with frequency $\omega$. Over a sample of $N$ points $\left(P_{1}, \ldots, P_{N}\right)$ we denote by $P_{n_{1}}$ the nearest neighbor to $P_{0}$ and we define the integer $p_{1}$ through the expression $n_{1} \omega=p_{1}+\epsilon_{1}$, where $\epsilon_{1}$ is a small quantity. Since $p_{1}$ counts the number of revolutions performed around the invariant curve, the quantity $\omega$ can be approximated by the ratio $p_{1} / n_{1}$. Increasing $N$, one gets a sequence of better approximations $p_{k} / n_{k}$ converging to $\omega$ up to small errors $\epsilon_{k}$. Particular care must be taken when applying this method to the dissipative case, since the starting point must be close to the attractor; to this end, a preliminary set of iterations, typically $10^{4}$, is performed before defining the starting point $P_{0}$.

In order to investigate the effect of the joined variation of the dissipative and of the perturbing parameters, we use frequency analysis by drawing the curve $\omega=\omega(b)$ for different values of $\varepsilon$ (see Figure 3 ). This approach allows to recognize the different kinds of attractors: indeed, invariant curves are characterized by a monotone variation of the frequency curve, periodic orbits show a marked plateau, while strange attractors exhibit an irregular behavior of the function $\omega=\omega(b)$. From experiments on different mappings and different choices of $\alpha$, we notice that invariant curves typically occur more frequently for small values of $\varepsilon$, while periodic and strange attractors appear more often as $\varepsilon$ gets larger. With reference to Figure 3, we also remark that Figure $3 a$ is ruled by the irrational choice of $\alpha$, while in Figure $3 b$ there is a dominant periodic attractor with period $\frac{1}{3}$ whose basin of attraction increases as the parameter $\varepsilon$ gets larger. The remaining panels refer to the two-frequency map $s_{1,3}(2 \pi x)$; in Figure $3 c$ the irrational choice of $\alpha$ is compensated by the selection of the harmonics 1 and 3 which appear in the mapping $s_{1,3}(2 \pi x)$, while in Figure $3 d$ both the choice of $s_{1,3}(2 \pi x)$ and of $\alpha$ induce most of the orbits to be attracted by the periodic attractor with frequency $\frac{1}{3}$.

\subsection{Differential Fast Lyapunov Indicator}

A global analysis of conservative systems can be performed through the Fast Lyapunov Indicators (hereafter, FLI) which are defined as follows. Let $\tilde{M}$ be the lift of the mapping; let us denote by $z(0) \equiv(y(0), x(0))$ the initial condition and let $v(0) \equiv\left(v_{y}(0), v_{x}(0)\right)$ be an initial vector with unitary norm. For a fixed time $T>0$, define the FLI as the quantity

$$
\overline{F L I}(z(0), v(0), T) \equiv \sup _{0<t \leq T} \log \|v(t)\|,
$$

where $v(t)$ is the solution of the differential system

$$
\left\{\begin{array}{l}
z(t+1)=\tilde{M}(z(t)) \\
v(t+1)=\frac{\partial \tilde{M}}{\partial z}(z(t)) v(t)
\end{array}\right.
$$

with initial data $z(0), v(0)$. We stress that in the unperturbed case $(\varepsilon=0)$, the largest Lyapunov exponent of an invariant curve is zero, while in the 
a)
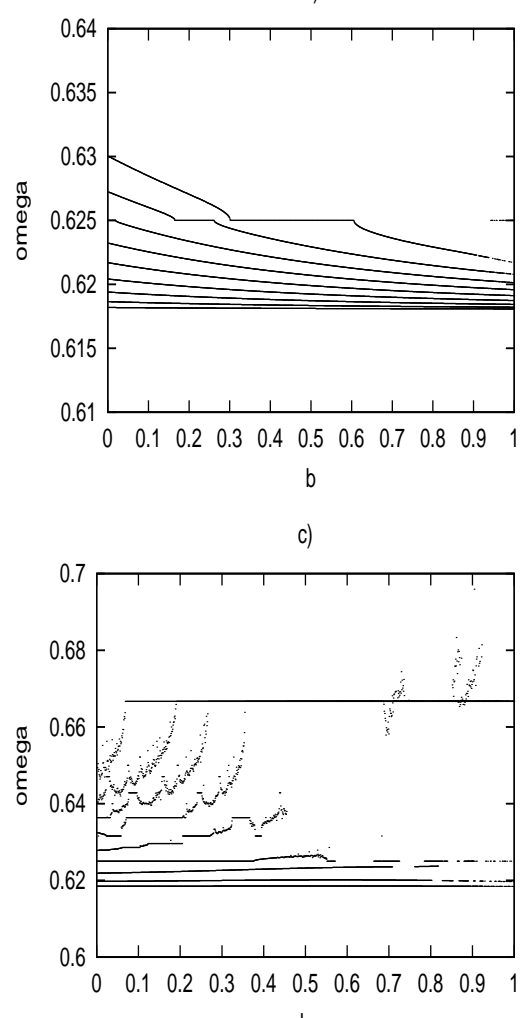

b)

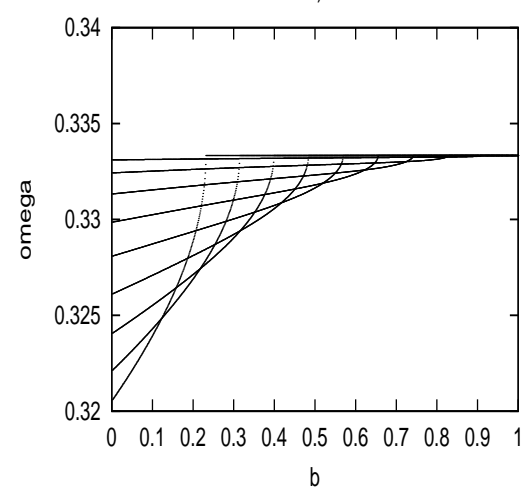

d)

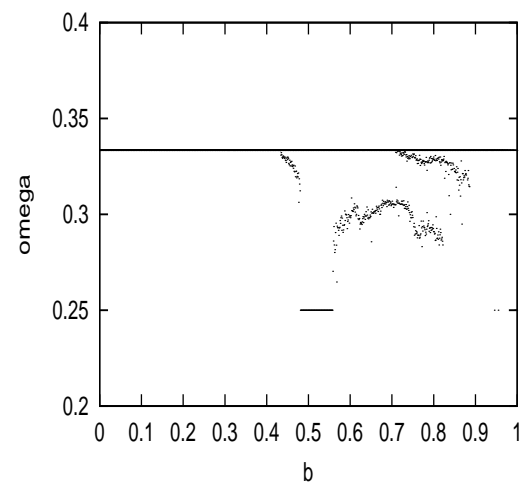

Fig. 3. Frequency analysis showing $\omega=\omega(b)$ for 9 different values of $\varepsilon$ from $\varepsilon=0.1$ (lower curve) to $\varepsilon=0.9$ (upper curve). a) Mapping $s_{1}(2 \pi x)$ with $\alpha=\frac{\sqrt{5}-1}{2} ; b$ ) Mapping $s_{1}(2 \pi x)$ with $\left.\alpha=\frac{1}{3} ; c\right)$ Mapping $s_{1,3}(2 \pi x)$ with $\left.\alpha=\frac{\sqrt{5}-1}{2} ; d\right)$ Mapping $s_{1,3}(2 \pi x)$ with $\alpha=\frac{1}{3}$ (after [8]).

dissipative case the corresponding $F L I$ can take any value within the range $\left[\log \left(\left|v_{x}(0)\right|\right),+\infty\right)$. By continuity the same problem holds for $\varepsilon \neq 0$; as a consequence, in the dissipative setting the FLI might not be adequate to differentiate between an invariant curve attractor and a strange attractor. Henceforth we defined in [8] the quantity

$$
D F L I_{0}(z(0), v(0), t) \equiv F(z(0), v(0), 2 t)-F(z(0), v(0), t),
$$

where $F(z(0), v(0), t)=F(t) \equiv \log \|v(t)\|$. We remark that $D F L I_{0}$ is zero for curve attractors, negative for periodic orbits and positive for chaotic attractors, in agreement with the value of the corresponding largest Lyapunov exponent. Finally, in order to kill the oscillations of the norm of the vector $v$ a supremum has been introduced, which corresponds to adopt the following 
definition of differential FLI:

$$
D F L I(T)=G_{2 T}(F(t))-G_{T}(F(t)),
$$

where

$$
\begin{cases}G_{\tau}(F(t))=\sup _{0 \leq t \leq \tau} F(t) & \text { if } F(\tau) \geq 0 \\ G_{\tau}(F(t))=\inf _{0 \leq t \leq \tau} F(t) & \text { if } F(\tau)<0 .\end{cases}
$$

The DFLI provides a complementary investigation to frequency analysis; to represent it in an effective way, we used a color scale which helps to discriminate among the different attractors. As performed in [8] we computed grids of $500 \times 500$ initial values of $b$ and $\varepsilon$ regularly spaced in the interval [0.01:1]; the initial conditions were set to $y_{0}=5$ and $x_{0}=0$, while $T=10^{3}$ (see (2)), after a transient of $10^{4}$ iterations. Then, the color classification is performed on the following basis: invariant curve attractors are denoted by grey and their $D F L I$ values are close to zero; strange attractors are labeled by light grey and their DFLI values are positive; periodic orbit attractors are denoted by dark grey to black with a negative DFLI.

As an example, we consider the two-frequency mapping $s_{1,3}(2 \pi x)$ with $\alpha=$ $\frac{\sqrt{5}-1}{2}$; the results are presented in Figure 4 . The left panel shows the chart of parameters $b$ versus $\varepsilon$ : scanning in the $\varepsilon$-direction we find invariant attractors up to $\varepsilon \simeq 0.36$ and periodic attractors around $\varepsilon \simeq 0.4$; for $\varepsilon>0.4$ a wide zone filled by periodic attractors is surrounded by two regions of strange attractors. The right panel provides the DFLI chart in the plane $b$ versus the initial condition $y$. We remark that for a fixed $b$, the basin of attraction is typically unique, with the exception of the parameter region $0.65<b<0.9$, where different initial conditions can be attracted either by a periodic orbit or by a strange attractor.
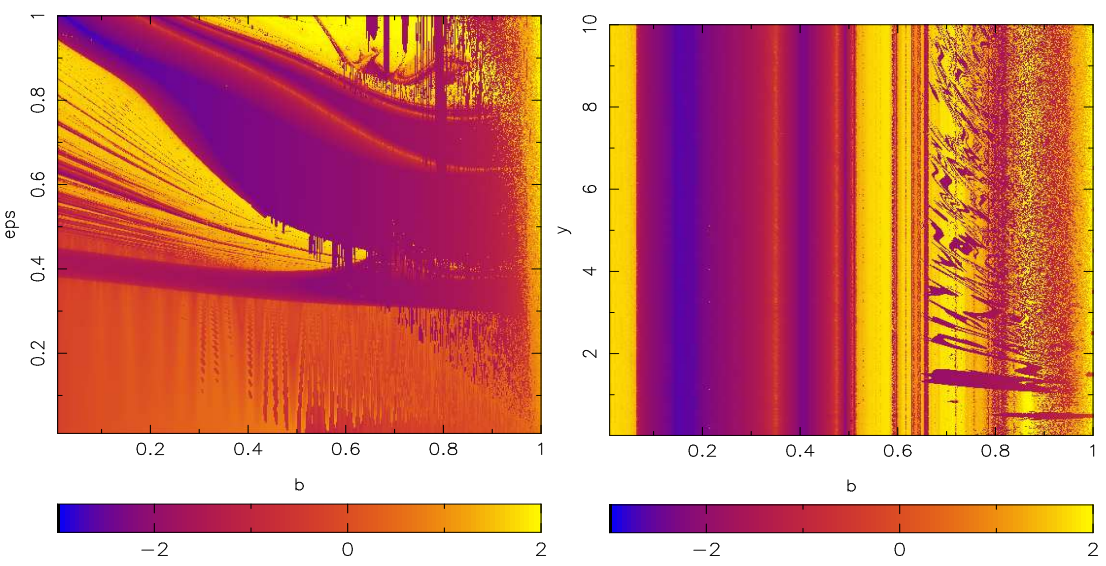

Fig. 4. Map $s_{1,3}(2 \pi x)$ with $\alpha=\frac{\sqrt{5}-1}{2}$. Left panel: DFLI chart $b$ vs. $\varepsilon$; right: DFLI chart $b$ vs. $y$ (after [8]). 
An important issue, especially from the point of view of physical applications, is the occurrence of periodic orbits and precisely the dependence of a given periodic attractor upon the choice of $\alpha$ and of the mapping $s(x)$. Numerical experiments (see [8]) show that a $q$-periodic orbit is highly likely whenever $\alpha=\frac{p}{q}$ or $s(2 \pi x)=\sin (2 \pi x \cdot q)$. We remark also that periodic orbit attractors with small period occur more frequently and that new periodic orbits arise for increasing $b$.

The applications concerning Celestial Mechanics that we shall consider in the following sections are typically characterized by a small value of the dissipation $b$ when compared to the perturbing parameter $\varepsilon$. In view of such investigations we concentrate on the weakly dissipative regime, where $b$ varies in the interval $[0.9,1]$. To be concrete, let us consider the mapping $s(2 \pi x)=\sin (3 \cdot 2 \pi x)$ with $\alpha=\frac{1}{2}$ and let us count the number of occurrences of a periodic orbit attractor of period $q$ as $\varepsilon$ varies. This result is presented in Figure $5 a$ using a semi- $\log$ scale: the rotation number is computed taking 100 initial conditions, say $x_{0}=0$ and $y_{0}$ in the interval $[0,10)$ and 1000 values of $b$ in [0.901, 0.999], while $\varepsilon$ takes the discrete values $0.1,0.2, \ldots, 0.9$.
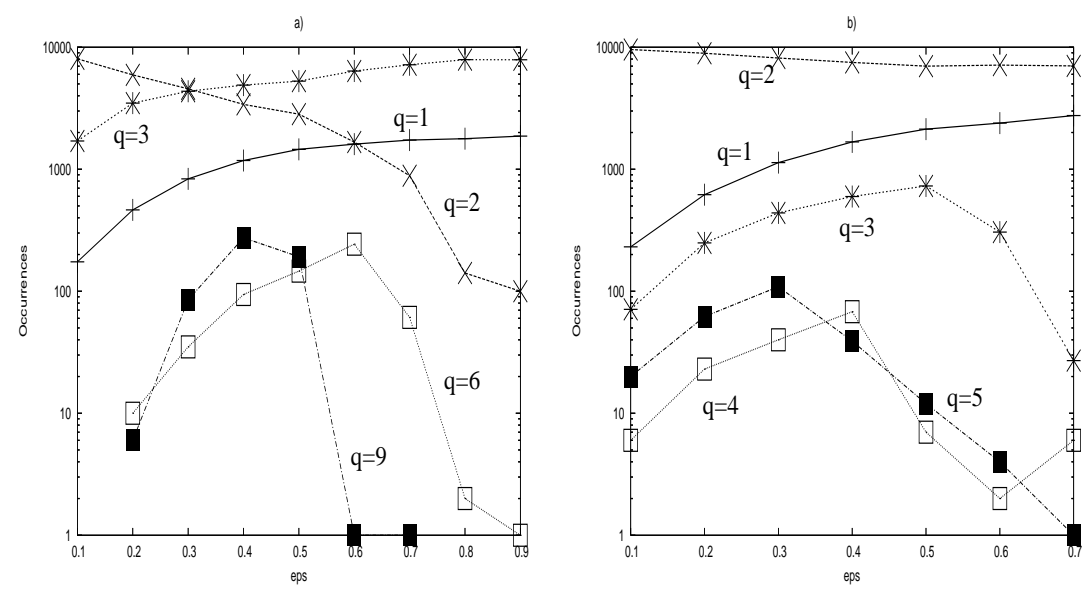

Fig. 5. Occurrence of periodic attractors versus $\varepsilon$. a) Mapping $s(2 \pi x)=\sin (3 \cdot 2 \pi x)$ with $\left.\alpha=\frac{1}{2} ; b\right)$ mapping $s(2 \pi x)=\frac{\sin (2 \pi x)}{\cos (2 \pi x)+1.4}$ with $\alpha=\frac{1}{2}$ (after [8]).

This experiment shows that there is a competition between the frequency $q=3$ (equal to the leading harmonic of $s(2 \pi x)$ ) and the frequency $q=2$ (as a consequence of the choice $\alpha=\frac{1}{2}$ ). The occurrence of periodic orbits with period 3 increases as $\varepsilon$ gets larger; on the other hand, the occurrence of the frequency $q=2$ increases as $\varepsilon$ gets smaller, which means that $\alpha$ is dominant for low values of $\varepsilon$. This example contributes to explain the roles of $\alpha$ and $s(2 \pi x)$ 
in the weakly dissipative regime. In a similar way we interpret the results for the case of the mapping $s(2 \pi x)=\frac{\sin (2 \pi x)}{\cos (2 \pi x)+1.4}$ which admits a full Fourier spectrum (Figure $5 b$ ). We remark that the weakly dissipative solution can be analyzed also perturbatively by introducing the small quantity $\beta \equiv 1-b$. Indeed, let us develop the solution in powers of $\beta$ as $y=y^{(0)}+\beta y^{(1)}+\beta^{2} y^{(2)}+$ $\ldots, x=x^{(0)}+\beta x^{(1)}+\beta^{2} x^{(2)}+\ldots$; inserting such equations in the definition of the mapping, one easily gets recursive relations on the quantities $y^{(k)}, x^{(k)}$. The investigation of these series expansion might provide information about the solution in the weakly dissipative regime.

\subsection{The 4-dimensional standard mapping}

The results presented for the two-dimensional mapping can be easily generalized to higher dimensional maps as well as to continuous systems. For example, let us consider the dissipative 4-dimensional standard map described by the equations

$$
\begin{aligned}
& y^{\prime}=b y+c_{1}+\varepsilon[\sin (x)+\gamma \sin (x-t)] \\
& x^{\prime}=x+y^{\prime} \\
& z^{\prime}=b z+c_{2}+\varepsilon[\sin (t)-\gamma \sin (x-t)] \\
& t^{\prime}=t+z^{\prime}
\end{aligned}
$$

where $y, z \in \mathbf{R}, x, t \in[0,2 \pi)$ and $c_{1}, c_{2}$ are real constants. The mapping depends also on three parameters: $b \in \mathbf{R}_{+}$is the dissipative parameter, $\varepsilon \in \mathbf{R}_{+}$ is the perturbing parameter, $\gamma \in \mathbf{R}_{+}$is the coupling parameter. Indeed, for $\gamma=0$ we obtain two uncoupled 2-dimensional standard mappings; we also remark that for $\varepsilon=0$ we obtain two uncoupled mappings which admit rotational invariant circles with frequencies $\alpha_{1} \equiv \frac{c_{1}}{1-b}$ and $\alpha_{2} \equiv \frac{c_{2}}{1-b}$. Let $\boldsymbol{\omega}=\left(\omega_{1}, \omega_{2}\right)$ be the frequency vector. With reference to Figure 6 we select $\boldsymbol{\omega}=\left(\frac{1}{s}, s-1\right)=(0.754877 \ldots, 0.324717 \ldots), s$ being the root of the third order polynomial $s^{3}-s-1=0$ (i.e., the smallest Pisot-Vijayaraghavan number of third degree; see, e.g., [7]). Figure 6 shows the two main frequencies as a function of the perturbing parameter in the case $b=0.7, \gamma=0.8$. A regular behavior is observed for values of $\varepsilon \leq 0.5$, followed by a chaotic motion manifested by an irregular variation of the frequency curves.

\section{Adiabatic invariants of the pendulum}

Let us consider a pendulum equation to which we add a small linear dissipative force, say

$$
\ddot{x}+\alpha \sin x+\beta \dot{x}-\gamma=0,
$$

for $x \in[0,2 \pi]$. We can write the above equation also as 


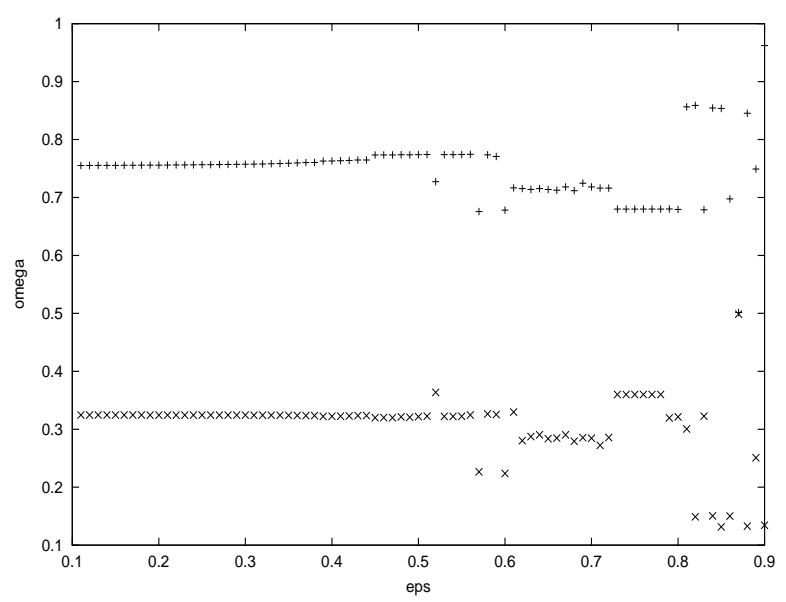

Fig. 6. Frequency analysis of the 4-dimensional standard map with $\boldsymbol{\omega}=$ $(0.754877 \ldots, 0.324717 \ldots), b=0.7, \gamma=0.8$ and initial conditions $y=1, x=0$, $z=0.7, t=0$.

$$
\begin{aligned}
& \dot{y}=-\alpha \sin x-\beta y \\
& \dot{x}=y-\frac{\gamma}{\beta} ;
\end{aligned}
$$

we remark that the choice of this example is motivated by the fact that it is very close to the spin-orbit equation described in the following section.

According to [18] the adiabatic invariant $Y \equiv \frac{1}{2 \pi} \oint y d x$ slowly changes for a small variation of the dissipation factor $\beta$ according to $Y(t)=e^{-\beta t} Y(0)$. The phase-space area enclosed by a guiding trajectory is provided by the formula

$$
\Gamma \equiv \oint \dot{x} d x=2 \pi Y-\frac{\gamma}{\beta} \oint d x
$$

As shown in [18], in case of positive circulation the spin slows approaching the resonance; in the librational regime the trajectory tends to the exact resonance; for negative circulation there are two possible behaviors: if $8 \sqrt{\alpha}>$ $2 \pi \frac{\gamma}{\beta}$ the guiding trajectory tends to the resonance, while if $8 \sqrt{\alpha}<2 \pi \frac{\gamma}{\beta}$ the motion can evolve toward an invariant curve attractor.

To provide a concrete example, let us follow the trajectory with initial conditions $x=0, y=-0.2$; the set of parameters $(\alpha, \beta, \gamma)=(0.0061,0.01,0.001)$ satisfies the condition $8 \sqrt{\alpha}<2 \pi \frac{\gamma}{\beta}$ and the corresponding dynamics is attracted by an invariant curve (see Figure 7 , left panel); on the contrary, such condition is not fulfilled by $(\alpha, \beta, \gamma)=(0.0063,0.01,0.001)$ and consistently we find that the corresponding trajectory is attracted by a resonance as shown in Figure 7, right panel. 

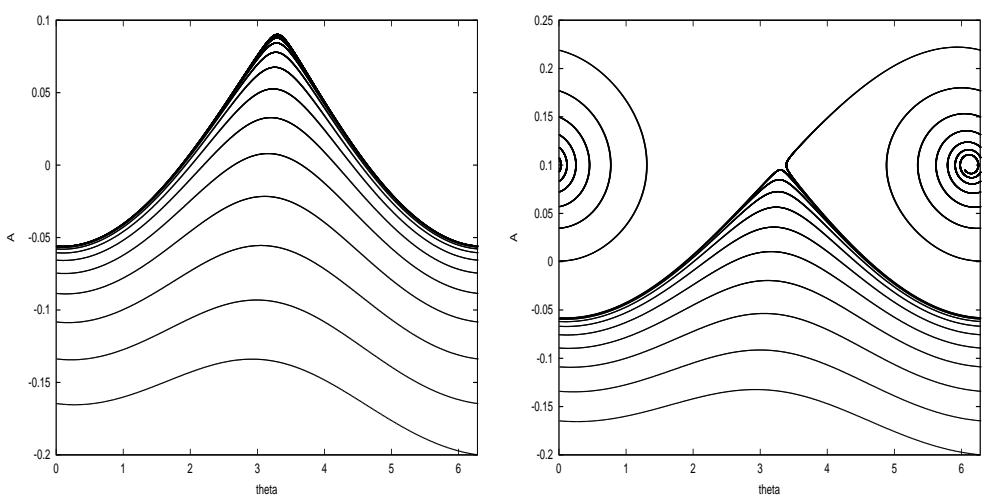

Fig. 7. Evolution of the dissipative pendulum in the phase space; left panel: attraction to an invariant curve, right panel: approach to a resonance.

\section{A paradigm from Celestial Mechanics: the spin-orbit model}

\subsection{The conservative model}

A simple interesting physical problem which gathers together many features of nearly-integrable weakly dissipative systems is provided by the spin-orbit coupling in Celestial Mechanics. Let us start with the description of the conservative model. We immediately remark that under suitable assumptions the equation of motion describing such model is very similar to the pendulum equation already met in the context of adiabatic invariants. More precisely, the model is the following: we consider a triaxial satellite $S$ orbiting around a central planet $P$ and rotating at the same time about an internal spin-axis. We denote by $T_{\text {rev }}$ and $T_{\text {rot }}$ the periods of revolution and rotation of $S$. The Solar System provides many examples of the so-called spin-orbit resonances, which are characterized by peculiar relationships between the revolution and rotation periods, according to the following

Definition. A spin-orbit resonance of order $p: q\left(\right.$ with $\left.p, q \in \mathbf{Z}_{+}, q \neq 0\right)$ occurs whenever

$$
\frac{T_{\text {rev }}}{T_{\text {rot }}}=\frac{p}{q} .
$$

In order to write the equations of motion, we make the following hypotheses:

i) the satellite moves on a Keplerian orbit around the planet;

ii) the spin-axis coincides with the smallest physical axis of the ellipsoid;

iii) the spin-axis is perpendicular to the orbital plane.

Under these assumption the equation of motion can be written as follows. Let $a, r, f$ be the semimajor axis, the instantaneous orbital radius and the true anomaly of the satellite; let $A<B<C$ be its principal moments of 
inertia and let $x$ be the angle between the longest axis of the satellite and the pericentre line. Then, the motion is described by the equation

$$
\ddot{x}+\frac{3}{2} \frac{B-A}{C}\left(\frac{a}{r}\right)^{3} \sin (2 x-2 f)=0 .
$$

Notice that the quantities $r$ and $f$ are known Keplerian functions of the time; setting $\varepsilon \equiv \frac{3}{2} \frac{B-A}{C}$, equation (3) can be expanded in Fourier series as (see, e.g. $[5])$

$$
\ddot{x}+\varepsilon \sum_{m=-\infty, m \neq 0}^{\infty} W(m, e) \sin (2 x-m t)=0,
$$

for some coefficients $W(m, e)$ which decay as powers of the eccentricity being proportional to $e^{|2 m-2|}$.

We remark that the parameter $\varepsilon$ represents the equatorial oblateness of the satellite; when $\varepsilon=0$ one has equatorial symmetry and the equation of motion is trivially integrable. Moreover, the dynamical system is integrable also in the case of circular orbit, since the radius $r$ coincides with the semimajor axis and the true anomaly becomes a linear function of the time.

\subsection{The dissipative model}

In writing equation (3) (equivalently (4)) we have neglected many contributions, like the gravitational attraction due to other celestial bodies or any kind of dissipative forces. Among the dissipative terms, the strongest contribution is due to the internal non-rigidity of the satellite. This tidal torque may assume different mathematical formulations; among the others we quote the classical MacDonald's ([24]) and Darwin's ([11]) torques which are reviewed in the following subsections (see [16], [26]). Let us summarize by saying that MacDonald expression assumes a phase lag depending linearly on the angular velocity, while Darwin's formulation Fourier decomposes the tidal potential, assigning to each component a constant amplitude. For more elaborated formulations of the tidal torque involving the internal structure of the satellite we refer to [19].

\section{MacDonald's torque}

Let $\delta$ be the angle formed by the direction to the planet and the direction to the maximum of the tidal bulge. Let us denote by $\underline{\hat{r}}$ the versor to $P$ and by $\underline{\hat{r}}_{T}$ the versor to the tidal maximum, i.e. the sub-planet position on $S$ a short time, say $\Delta t$, in the past; then we have

$$
\underline{\hat{\hat{r}}}_{T}=\underline{\hat{\hat{r}}}(t-\Delta t) \simeq \underline{\hat{\hat{r}}}-\frac{d \underline{\hat{r}}}{d t} \Delta t,
$$

where the derivative is computed in the body-frame. Using the relations $\cos \delta=\underline{\hat{r}} \cdot \underline{\hat{r}}_{T}, \sin \delta=\underline{\hat{\hat{r}}}_{T} \wedge \underline{\hat{\hat{r}}}$, we obtain that MacDonald's torque takes the expression (see, e.g., [16]) 


$$
\begin{aligned}
\underline{T} & =\frac{3 k_{2} G m_{P}^{2} R^{5}}{2 r^{6}} \sin (2 \delta) \\
& =\frac{3 k_{2} G m_{P}^{2} R^{5}}{r^{6}}\left(\underline{\hat{r}} \cdot \underline{\hat{r}}_{T}\right)\left(\underline{\hat{r}}_{T} \wedge \underline{\hat{r}}\right),
\end{aligned}
$$

where $k_{2}$ is the so-called Love's number, $G$ is the gravitational constant, $m_{P}$ is the mass of the planet, $R$ is the satellite's mean radius. Taking into account (5) and the relation $\underline{\hat{r}} \cdot \underline{\hat{r}}_{T}=1$, we obtain

$$
\underline{T}=\frac{3 k_{2} G m_{P}^{2} R^{5} \Delta t}{r^{6}}\left(\underline{\hat{r}} \wedge \frac{d}{d t} \underline{\hat{r}}\right) .
$$

Let $\left(\underline{e}_{x}, \underline{e}_{y}, \underline{e}_{z}\right)$ be the versors of the reference orbital plane; denote by $\Omega$ the longitude of the ascending node, $I$ is the obliquity, while $\psi_{m}$ is the angle between the ascending node and the body axis of minimum moment of inertia. Then, one obtains the relation $([26])$

$$
\begin{aligned}
\underline{\hat{r}} \wedge \frac{d}{d t} \hat{\underline{r}} & =\dot{\psi}_{m} \sin I \cos (f-\Omega)\left(-\sin f \underline{e}_{x}+\cos f \underline{e}_{y}\right) \\
& +\left(\dot{f}-\dot{\Omega}-\dot{\psi}_{m} \cos I\right) \underline{e}_{z} .
\end{aligned}
$$

Taking only the component along the $z$-axis, one obtains that the average of the tidal torque over the orbital period is given by

$$
\langle\underline{T}\rangle=\frac{3 k_{2} G m_{P}^{2} R^{5} \Delta t}{a^{6}}\left(n N(e)-L(e) \dot{\psi}_{m} \cos I\right) \underline{e}_{z},
$$

where $n$ is the mean motion and $N(e), L(e)$ are related to the following averages over short-period terms:

$$
\begin{aligned}
& \left\langle\frac{a^{6}}{r^{6}} \dot{f}\right\rangle \equiv n N(e)=n\left(1+\frac{15}{2} e^{2}+\frac{45}{8} e^{4}+\frac{5}{16} e^{6}\right) \frac{1}{\left(1-e^{2}\right)^{6}} \\
& \left\langle\frac{a^{6}}{r^{6}}\right\rangle \equiv L(e)=\left(1+3 e^{2}+\frac{3}{8} e^{4}\right) \frac{1}{\left(1-e^{2}\right)^{9 / 2}} .
\end{aligned}
$$

According to assumption $\mathrm{iii}$ ) of the previous section we can set $I=0$, so that $\psi_{m}$ coincides with $x$. Finally, the equation of motion (3) under the effect of the MacDonald's torque is given by

$$
\ddot{x}+\frac{3}{2} \frac{B-A}{C}\left(\frac{a}{r}\right)^{3} \sin (2 x-2 f)=-K[L(e) \dot{x}-N(e)],
$$

where we have used $\omega \Delta t=\frac{1}{Q}, Q$ being the so-called quality factor ([26]), and where we have introduced a dissipation constant $K$ depending on the physical and orbital characteristics of the satellite:

$$
K \equiv 3 n \frac{k_{2}}{\xi Q}\left(\frac{R}{a}\right)^{3} \frac{m_{P}}{m_{S}},
$$


where $m_{S}$ is the mass of the satellite and $\xi$ is a structure constant such that $C=\xi m R^{2}$. For the cases of the Moon and Mercury the explicit values of such constants are given in Table I; it results that the dissipation constant amounts to $K=6.43162 \cdot 10^{-7} y r^{-1}$ for the Moon and $K=8.4687 \cdot 10^{-7} y^{-1}$ for Mercury.

Table I.

\begin{tabular}{lll}
\hline \multicolumn{1}{c}{ Moon } & Mercury \\
\hline \hline$k_{2}$ & 0.02 & 0.4 \\
$Q$ & 150 & 50 \\
$m_{S}$ & $7.35 \cdot 10^{22} \mathrm{~kg}$ & $3.302 \cdot 10^{23} \mathrm{~kg}$ \\
$m_{P}$ & $5.972 \cdot 10^{24} \mathrm{~kg}$ & $1.99 \cdot 10^{30} \mathrm{~kg}$ \\
$R$ & $1737.5 \mathrm{~km}$ & $2440 \mathrm{~km}$ \\
$\xi$ & 0.392 & 0.333 \\
$a$ & $3.844 \cdot 10^{5} \mathrm{~km}$ & $5.79093 \cdot 10^{7} \mathrm{~km}$ \\
$e$ & 0.0554 & 0.2056 \\
$n$ & $84.002 \mathrm{yr}^{-1}$ & $26.0879 \mathrm{yr}^{-1}$ \\
$K$ & $6.43162 \cdot 10^{-7} \mathrm{yr}^{-1} 8.4687 \cdot 10^{-7} \mathrm{yr}^{-1}$ \\
\hline
\end{tabular}

\section{Darwin's torque}

In the case of Darwin's torque we just provide the explicit expression which is related to the Fourier expansion (4) as

$$
\begin{aligned}
& \quad \ddot{x}+\frac{3}{2} \frac{B-A}{C}\left(\frac{a}{r}\right)^{3} \sin (2 x-2 f)=-K\left(W(-2, e)^{2} \operatorname{sgn}(x+1)\right. \\
& +W(-1, e)^{2} \operatorname{sgn}\left(x+\frac{1}{2}\right)+W(1, e)^{2} \operatorname{sgn}\left(x-\frac{1}{2}\right)+W(2, e)^{2} \operatorname{sgn}(x-1) \\
& +W(3, e)^{2} \operatorname{sgn}\left(x-\frac{3}{2}\right)+W(4, e)^{2} \operatorname{sgn}(x-2)+W(5, e)^{2} \operatorname{sgn}\left(x-\frac{5}{2}\right) \\
& \left.+W(6, e)^{2} \operatorname{sgn}(x-3)\right)
\end{aligned}
$$

where the coefficients $W_{k}$ take the form

$$
\begin{aligned}
W(-2, e) & =\frac{e^{4}}{24} & W(-2, e) & =\frac{e^{3}}{48} \\
W(1, e) & =-\frac{e}{2}+\frac{e^{3}}{16} & W(2, e) & =1-\frac{5 e^{2}}{2}+\frac{13 e^{4}}{16} \\
W(3, e) & =\frac{7 e}{2}-\frac{123 e^{3}}{16} & W(4, e) & =\frac{17 e^{2}}{2}-\frac{115 e^{4}}{6} \\
W(5, e) & =\frac{845 e^{3}}{48} & W(6, e) & =\frac{533 e^{4}}{16} .
\end{aligned}
$$




\subsection{Capture into resonance}

Bearing in mind the discussions about the capture into resonance for the dissipative standard map, we proceed to illustrate some classical results (see [16], [27]) about resonance capture in the spin-orbit problem. We will see that such event strongly depends on the form of the dissipation. Using (4) let us write the dissipative spin-orbit equation as

$$
\ddot{x}+\frac{3}{2} \frac{B-A}{C} \sum_{m=-\infty, m \neq 0}^{\infty} W(m, e) \sin (2 x-m t)=T .
$$

Let us introduce the $p$-resonant angle $\gamma \equiv x-p t$; after averaging over one orbital period one gets

$$
C \ddot{\gamma}+\frac{3}{2}(B-A) W(p, e) \sin 2 \gamma=\langle T\rangle .
$$

\section{Constant torque}

Let us consider the case $\langle T\rangle=$ const; a first integral associated to (7) is trivially obtained as

$$
\frac{1}{2} C \dot{\gamma}^{2}-\frac{3}{4}(B-A) W(p, e) \cos 2 \gamma=\langle T\rangle \gamma+E_{0} \equiv E .
$$

We plot in Figure 8 the behavior of $\frac{1}{2} \dot{\gamma}^{2}$ versus $\gamma$ as derived from (8) (see [16]). We denote by $\gamma_{\max }$ the point at which $\dot{\gamma}=0$. Assuming an initial positive $\dot{\gamma}$, we proceed along the curve until we reach $\gamma_{\max }$; at this moment the motion reverses sign, thus escaping from the resonance.

\section{MacDonald's case}

Let us now assume that $\langle T\rangle=-K_{1}(\dot{\gamma}+V)$ for some constants $K_{1}$ and $V$; then we have:

$$
\frac{d}{d t}\left[\frac{1}{2} C \dot{\gamma}^{2}-\frac{3}{4}(B-A) W(p, e) \cos 2 \gamma\right]=-K_{1}\left(\dot{\gamma}^{2}+V \dot{\gamma}\right)=\frac{d E}{d t},
$$

where the behavior of $\frac{1}{2} \dot{\gamma}^{2}$ is provided in Figure 9. Let us denote by $\Delta E$ the difference of $\frac{\dot{\gamma}^{2}}{2}$ between two successive minima, say $\gamma_{1}$ and $\gamma_{2}$; let $\delta E$ be the difference between the two minima with the same ordinate $\gamma_{1}$. Let $E_{1}$ be the ordinate of the highest minimum at $\gamma_{1}$; being $|\delta E|>E_{1}$ there exists a second zero of $\frac{\dot{\gamma}^{2}}{2}$ near $\gamma_{1}$, so that $\dot{\gamma}$ reverses sign again and $\gamma$ is trapped in libration between $\gamma_{1}$ and $\gamma_{2}$.

On the other hand, if $|\delta E|<E_{1}$ the planet escapes from the resonance and continues to despin (see Figure 10). 


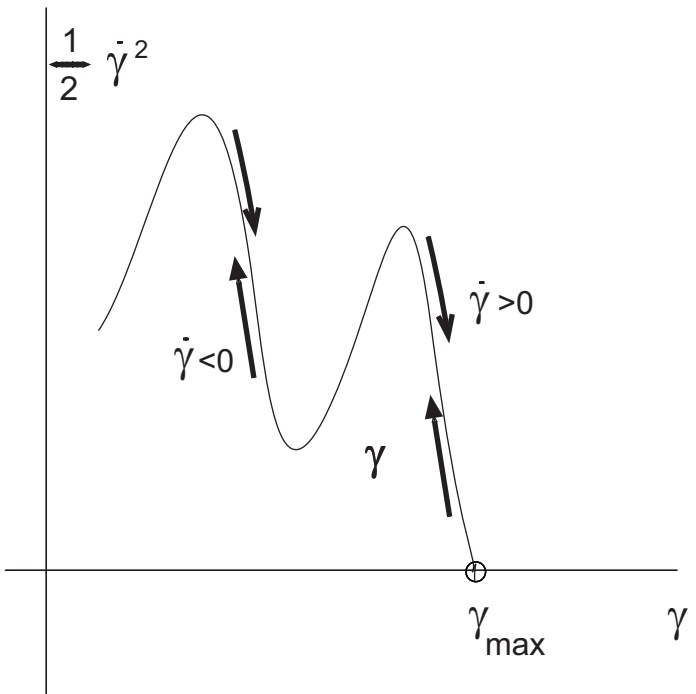

Fig. 8. Case $\langle T\rangle=$ const.

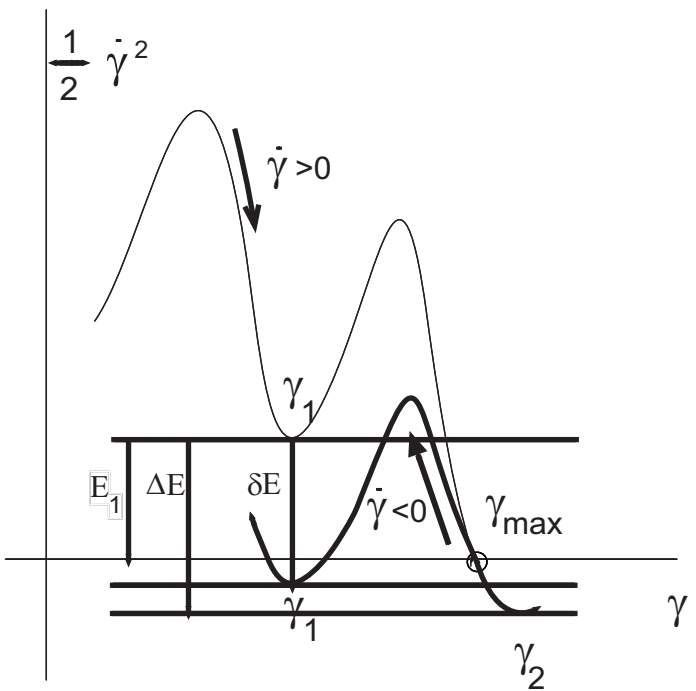

Fig. 9. MacDonald's case: trapping in libration. 


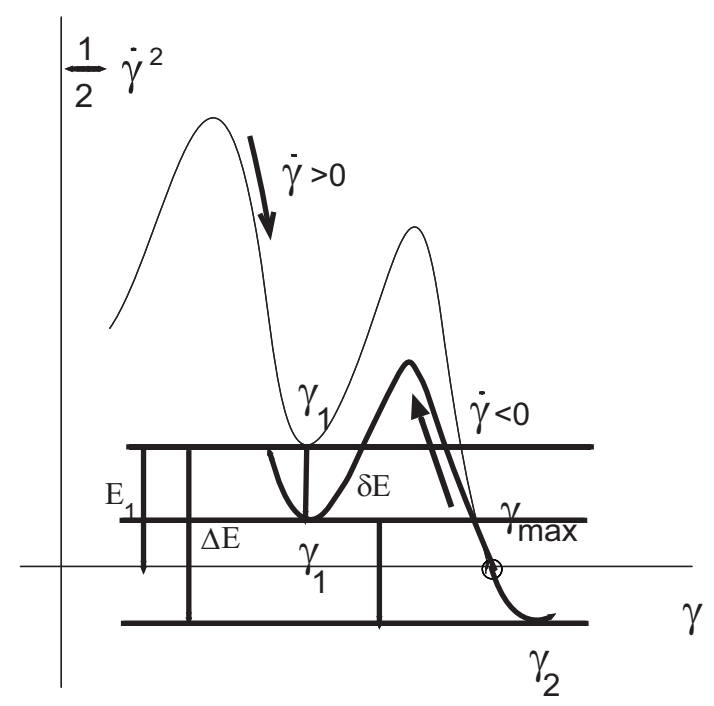

Fig. 10. MacDonald's case: escape from resonance.

Following [16] we can compute the probability of capture $P$ as follows. Assuming that the values of $E_{1}$ are distributed with uniform probability in $[0, \Delta E]$ we define $P$ as $P \equiv \frac{\delta E}{\Delta E}$; then we obtain

$$
P \simeq \frac{2}{1+\frac{\pi V}{\int_{\gamma_{1}}^{\gamma 2} \dot{\gamma} d \gamma}}
$$

\section{Darwin's case}

We assume that $\langle T\rangle=-W-Z \operatorname{sgn}(\dot{\gamma})$, for some constants $W$ and $Z$. Denote by $\delta E^{\prime}$ the difference of $\frac{1}{2} \dot{\gamma}^{2}$ between $\gamma_{2}$ and the second minimum at $\gamma_{1}$; then, one can easily show that (see [16])

$$
\Delta E=-(W+Z) \pi \quad \delta E=-2 \pi Z,
$$

so that the probability of capture can be written as

$$
P=\frac{2 Z}{W+Z}
$$

which turns out to be independent on $\frac{B-A}{C}$.

\section{The restricted, planar, circular, 3-body problem}

Another basic example of a dissipative nearly-integrable system in Celestial Mechanics is represented by gravitationally interacting bodies, subject to a 
dissipative force. Focussing our attention to the restricted, planar, circular, 3 -body problem, we can derive the equations of motion in a synodic reference frame as follows. We investigate the motion of a massless body $S$ under the influence of two primaries $P_{1}, P_{2}$ with masses $\mu$ and $1-\mu$; we assume that the motion of the primaries is circular around their common barycenter and that all bodies move on the same plane. If $\left(x, y, p_{x}, p_{y}\right)$ denote the coordinates of the minor body in the synodic frame, the equations of motion under a linear dissipation read as

$$
\begin{aligned}
\dot{x} & =y+p_{x} \\
\dot{y} & =-x+p_{y} \\
\dot{p}_{x} & =p_{y}-\frac{1-\mu}{r_{1}^{3}}(x+\mu)-\frac{\mu}{r_{2}^{3}}(x-1+\mu)-K p_{x} \\
\dot{p}_{y} & =-p_{x}-\frac{1-\mu}{r_{1}^{3}} y-\frac{\mu}{r_{2}^{3}} y-K p_{y},
\end{aligned}
$$

where $r_{1} \equiv \sqrt{(x+\mu)^{2}+y^{2}}, r_{2} \equiv \sqrt{(x-1+\mu)^{2}+y^{2}}$. The effect of the dissipation is simulated by adding the terms $\left(-K p_{x},-K p_{y}\right)$ to the equations for $\dot{p}_{x}$ and $\dot{p}_{y}$. Let us now look at the survival of periodic orbits under the effect of the dissipation. By Newton's method we determine the periodic orbit in the conservative case; then we slowly increase the dissipation parameter and we compute the periodic orbit through a continuation method. This procedure might fail or work in different situations as shown in Figure 11: the left panel provides a periodic orbit in the conservative setting with period twice the basic period of the primaries; however, such orbit immediately disappears as $K \neq 0$. On the other hand the periodic orbit shown in the right panel of Figure 11 has period 3 times the basic period of the primaries and can be continued in the dissipative context up to $K=10^{-4}$.

We conclude by mentioning that many other dissipative forces can be considered in the framework of the 3-body problem, such as the solar wind (which is caused by charged particles originating from the upper atmosphere of the Sun), the Yarkowsky effect (consisting in the anisotropic emission of thermal photons due to the rotation of the celestial body), the radiation pressure (caused by electromagnetic radiation). This last force is a component of the Poynting-Robertson drag exerted by solar radiation on dust grains. Other dissipative forces widely studied in the literature are the Stokes and Epstein drags, which affect the orbital evolution of a dust grain in a gas planetary nebula, and are respectively valid for low and high Reynold numbers.

\section{Periodic orbits for non-autonomous and autonomous systems}

A remarkable discussion of the existence of periodic orbits for non-autonomous and autonomous systems can be found in [27] (see also [28], [9], [30]). Indeed, 

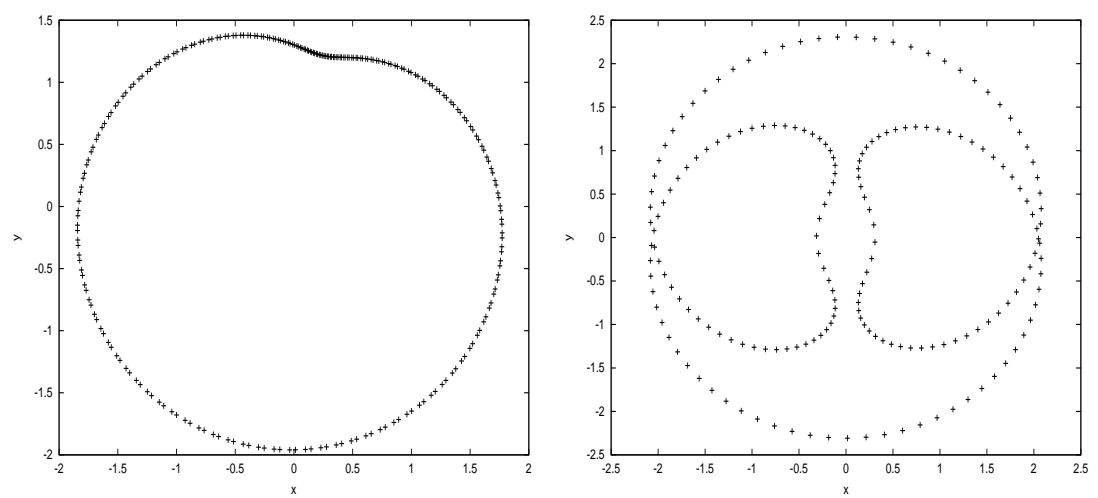

Fig. 11. Left panel: periodic orbit of period 2 for $K=0$, corresponding to a semimajor axis equal to 1.58 and eccentricity equal to 0.22 . Right panel: periodic orbit of period 3 for $K=0$, corresponding to a semimajor axis equal to 1.31 and eccentricity equal to 0.76 .

the arguments we are going to present apply to the two examples we have discussed so far, namely the non-autonomous spin-orbit problem and the autonomous restricted, planar, circular, 3-body problem. In particular, we want to show that

i) for the non-autonomous spin-orbit problem described by (6) one can find periodic orbits with period equal (or multiple) to that of the conservative problem;

ii) for the restricted, planar, circular, 3-body problem one can apply in the conservative case (eq.s (9) with $K=0$ ) the theory for autonomous systems, which allows to find periodic solutions with the same period of the case in which the perturbing parameter $\mu$ is set to zero;

iii) for the dissipative restricted, planar, circular, 3-body problem, one can use the autonomous theory to find periodic orbits with period close (but not exactly equal) to that obtained for $K=0$.

We report in the Appendix the perturbative computation up to the second order of periodic orbits in an autonomous dissipative case.

\subsection{Non-autonomous systems}

Consider the differential equation

$$
\dot{x}=f(x, t ; \gamma),
$$

where $x=\left(x_{1}, \ldots, x_{n}\right)$ and $f$ is a $T$-periodic function depending on a real parameter $\gamma$. Assume that for $\gamma=0$ we know a $T$-periodic orbit described by the equations

$$
x(t)=\varphi(t) \quad \text { with } \quad \varphi(T)=\varphi(0) .
$$


Then, if $\gamma$ is sufficiently small, one can prove the existence of a periodic solution with period $T$. In fact, assume that the initial data of the periodic orbit for $\gamma \neq 0$ are

$$
x(0)=\varphi(0)+\beta,
$$

where $\beta=\left(\beta_{1}, \ldots, \beta_{n}\right)$. After one period one has

$$
x(T)=\varphi(0)+\beta+\psi,
$$

where $\psi=\left(\psi_{1}, \ldots, \psi_{n}\right)$ are holomorphic functions in $\beta_{1}, \ldots, \beta_{n}, \gamma$. In order to prove the existence of a periodic solution, the following $n$ equations in the unknowns $\left(\beta_{1}, \ldots, \beta_{n}\right)$ must be satisfied:

$$
\psi=\left(\psi_{1}, \ldots, \psi_{n}\right)=0 .
$$

Applying the implicit function theorem, if $\gamma$ is sufficiently small and if the Jacobian of $\psi$ with respect to $\beta$ satisfies

$$
\left.\left(\frac{\partial \psi}{\partial \beta}\right)\right|_{\gamma=\beta=0} \neq 0
$$

then there exists $\beta=\beta(\gamma)$ such that $\beta(0)=0$ and there exists a $T$-periodic orbit provided $\gamma$ is sufficiently small.

\subsection{Autonomous systems}

Consider the autonomous system

$$
\dot{x}=f(x ; \gamma),
$$

where $x=\left(x_{1}, \ldots, x_{n}\right)$ and $f$ depends on the real parameter $\gamma$. Assume that for $\gamma=0$ we know a $T$-periodic orbit, given by the equations

$$
x(t)=\varphi(t) \quad \text { with } \quad \varphi(T)=\varphi(0) .
$$

We immediately remark that if there is one periodic orbit, then there exists an infinity, since if $x(t)=\varphi(t)$ is periodic, also $x(t)=\varphi(t+h)$ for any real $h$ is periodic, being the system autonomous.

Let $\gamma \neq 0$ and look for a solution such that

$$
x(0)=\varphi(0)+\beta, \quad x(T+\tau)=\varphi(0)+\beta+\psi,
$$

where $\psi=\left(\psi_{1}, \ldots, \psi_{n}\right)$ are holomorphic in $\beta_{1}, \ldots, \beta_{n}, \gamma, \tau$.

Such solution is $(T+\tau)$-periodic if

$$
\psi=\left(\psi_{1}, \ldots, \psi_{n}\right)=0,
$$

which represents $n$ equations in the $n+1$ unknown quantities $\beta_{1}, \ldots, \beta_{n}, \tau$. 
Since there are several choices of the initial conditions which lead to the same orbit (indeed, taking any other point of the orbit we change only the epoch and not the orbit), we can arbitrarily set $\beta_{n}=0$. Therefore we have $n+1$ equations $\psi=\left(\psi_{1}, \ldots, \psi_{n}\right)=0, \beta_{n}=0$ in the $n+1$ unknowns $\beta_{1}, \ldots, \beta_{n}, \tau$. By the implicit function theorem, if the jacobian

$$
\left(\begin{array}{cccc}
\frac{\partial \psi_{1}}{\partial \beta_{1}} & \cdots & \frac{\partial \psi_{1}}{\partial \beta_{n-1}} & \frac{\partial \psi_{1}}{\partial \tau} \\
\vdots & & & \\
\frac{\partial \dot{\psi}_{n}}{\partial \beta_{1}} & \cdots & \frac{\partial \psi_{n}}{\partial \beta_{n-1}} & \frac{\partial \psi_{n}}{\partial \tau}
\end{array}\right)_{\gamma=\beta=\tau=0} \neq 0
$$

and if $\gamma$ is sufficiently small, there exists a periodic solution with period $T+\tau$.

\subsection{Autonomous systems with integrals}

In the autonomous case assume there exists an integral

$$
G(x)=C=\text { const }
$$

Then the equations

$$
\psi_{1}=\ldots=\psi_{n}=\beta_{n}=0
$$

are not anymore distinct and one can replace the above equations with

$$
\psi_{1}=\ldots=\psi_{n}=\beta_{n}=0, \quad G=C+\lambda \gamma,
$$

where $\lambda$ is a generic constant; alternatively, one can replace (10) with the equations

$$
\psi_{1}=\ldots=\psi_{n}=\beta_{n}=0, \quad \tau=0 .
$$

Equations (11) imply that the energy level is changed, while equations (12) imply that if there exists an integral $G(x)=C$, one can find a $T$-periodic solution for $\gamma$ small.

\section{A Second-order computation of periodic orbits in the autonomous dissipative case}

Consider the differential equations

$$
\dot{x}=f(x, \gamma),
$$

where $x=\left(x_{1}, \ldots, x_{n}\right)$ and $f$ depends also on a real small parameter $\gamma$. We want to look for a periodic solution with period $T_{\gamma}$, such that

$$
x\left(T_{\gamma}\right)=x(0) .
$$

To this end we expand the solution $x$ and the period $T_{\gamma}$ in series of $\gamma$ as 


$$
\begin{aligned}
x & =x_{0}+\gamma x_{1}+\gamma^{2} x_{2}+\ldots \\
T_{\gamma} & =T_{0}+\gamma T_{1}+\gamma^{2} T_{2}+\ldots
\end{aligned}
$$

Let us denote by $f_{\gamma}=\frac{\partial f}{\partial \gamma}, f^{\prime}=\frac{\partial f}{\partial x}, f^{\prime \prime}=\frac{\partial^{2} f}{\partial x^{2}}$; inserting (14) in (13) and equating same orders of $\gamma$ up to the 2 nd order we get

$$
\begin{aligned}
& \dot{x}_{0}=f\left(x_{0}, 0\right) \\
& \dot{x}_{1}=f^{\prime}\left(x_{0}, 0\right) x_{1}+f_{\gamma}\left(x_{0}, 0\right) \\
& \dot{x}_{2}=f^{\prime}\left(x_{0}, 0\right) x_{2}+\frac{1}{2} f^{\prime \prime}\left(x_{0}, 0\right) x_{1}^{2} .
\end{aligned}
$$

Suppose now that for $\gamma=0$ we know a $T_{0}$-periodic orbit, described by $x_{0}=$ $x_{0}(t)$ with initial data $x_{0}(0)$ and periodicity conditions $x_{0}\left(T_{0}\right)=x_{0}(0)$. Then, for $\gamma \neq 0$ we look for a $T_{\gamma}$-periodic orbit described by $x=x(t)$, whose initial data are displaced with respect to the conservative case as

$$
x(0)=x_{0}(0)+\beta ;
$$

moreover we require that the following periodicity conditions are satisfied:

$$
x\left(T_{\gamma}\right)=x_{0}(0)+\beta .
$$

Develop $\beta$ in powers of $\gamma$ as $\beta=\gamma \beta_{1}+\gamma^{2} \beta_{2}+\ldots$; from (15) one has

$$
x_{0}(0)+\gamma x_{1}(0)+\gamma^{2} x_{2}(0)+\ldots=x_{0}(0)+\gamma \beta_{1}+\gamma^{2} \beta_{2}+\ldots
$$

Comparing same orders of $\gamma$, one obtains $\beta_{j}=x_{j}(0)$, so that the $\beta_{j}$ 's are the corrections at order $j$ to the initial data. Using (16) and recalling that $x_{0}\left(T_{0}\right)=x_{0}(0)$, one obtains

$$
\begin{aligned}
& x_{0}\left(T_{0}\right)+\gamma \dot{x}_{0}\left(T_{0}\right)\left(T_{1}+\gamma T_{2}\right)+\frac{1}{2} \ddot{x}_{0}\left(T_{0}\right) \gamma^{2} T_{1}^{2}+x_{1}\left(T_{0}\right) \gamma+\dot{x}_{1}\left(T_{0}\right) T_{1} \gamma^{2}+x_{2}\left(T_{0}\right) \gamma^{2}+\ldots \\
& =x_{0}(0)+\gamma \beta_{1}+\gamma^{2} \beta_{2}+\ldots
\end{aligned}
$$

Equating same orders of $\gamma$ up to the order 2, one gets

$$
\begin{aligned}
\dot{x}_{0} & =f\left(x_{0}, 0\right) \\
x_{0}\left(T_{0}\right) & =x_{0}(0) \\
\dot{x}_{1} & =f^{\prime}\left(x_{0}, 0\right) x_{1}+f_{\gamma}\left(x_{0}, 0\right) \\
\beta_{1} & =x_{1}\left(T_{0}\right)+\dot{x}_{0}\left(T_{0}\right) T_{1} ; \\
\dot{x}_{2} & =f^{\prime}\left(x_{0}, 0\right) x_{2}+\frac{1}{2} f^{\prime \prime}\left(x_{0}, 0\right) x_{1}^{2} \\
\beta_{2} & =x_{2}\left(T_{0}\right)+\dot{x}_{0}\left(T_{0}\right) T_{2}+\frac{1}{2} \ddot{x}_{0}\left(T_{0}\right) T_{1}^{2}+\dot{x}_{1}\left(T_{0}\right) T_{1} .
\end{aligned}
$$

Let us analyze the last equation; recalling that $\beta_{2}=\left(\beta_{2}^{(1)}, \ldots, \beta_{2}^{(n)}\right)$, we have $n$ equations in the $n+1$ unknowns $\beta_{2}^{(1)}, \ldots, \beta_{2}^{(n)}, T_{2}$. To eliminate the ambiguity, we can set $\beta_{2}^{(n)}=0$ and solve the equations with respect to the remaining unknowns. 


\section{References}

1. C. Beaugé and S. Ferraz-Mello, Capture in exterior mean-motion resonances due to Poynting-Robertson drag, Icarus 110 (1994), 239-260.

2. C. Beaugé and S. Ferraz-Mello, Resonance trapping in the primordial solar nebula: The case of a Stokes drag dissipation, Icarus 103 (1993), 301-318.

3. T. Bohr, P. Bak and M.H. Jensen, Transition to chaos by interaction of resonances in dissipative systems. II. Josephson junctions, charge-density waves, and standard maps, Phys. Rev. A 30, n. 4 (1984), 1970-1981.

4. H.W. Broer, C. Simó and J.C. Tatjer, Towards global models near homoclinic tangencies of dissipative diffeomorphisms, Nonlinearity 11 (1998), 667-770.

5. A. Celletti, Analysis of resonances in the spin-orbit problem in Celestial Mechanics: The synchronous resonance (Part I), Journal of Applied Mathematics and Physics (ZAMP) 41 (1990), 174-204.

6. A. Celletti, G. Della Penna and C. Froeschlé, Analytical approximation of the solution of the dissipative standard map, Int. J. Bif. Chaos 8, n. 12 (1998), $2471-2479$.

7. A. Celletti, C. Falcolini and U. Locatelli, On the break-down threshold of invariant tori in four dimensional maps, Regular and Chaotic Dynamics 9, n. 3 (2004) 227-253.

8. A. Celletti, C. Froeschlé and E. Lega, Dissipative and weakly-dissipative regimes in nearly-integrable mappings, Discrete and Continuous Dynamical Systems Series A 16, n. 4, 757-781 (2006)

9. E.A. Coddington, and N. Levinson, Theory of ordinary differential equations, McGrawHill, New York (1995).

10. A.C.M. Correia, and J. Laskar, Mercury's capture into the 3/2 spin-orbit resonance as a result of its chaotic dynamics, Nature 429 (2004), 848-850.

11. G. Darwin, Tidal friction and cosmogony, Scientific papers, Cambridge University Press 2 (1908).

12. S. D'Hoedt, and A. Lemaitre, The Spin-Orbit Resonant Rotation of Mercury: A Two Degree of Freedom Hamiltonian Model, Cel. Mech. Dyn. Astr. 89, n. 3 (2004), 267-283.

13. C. Froeschlé, M. Guzzo and E. Lega, Graphical evolution of the Arnold's web: from order to chaos, Science 289, n. 5487 (2000), 2108-2110.

14. C. Froeschlé, E. Lega and R. Gonczi, Fast Lyapunov indicators. Application to asteroidal motion, Celest. Mech. and Dynam. Astron. 67 (1997), 41-62.

15. P. Goldreich, Final spin states of planets and satellites, Astronom. J. 71, n. 1 (1966), 1-7.

16. P. Goldreich, and S. Peale, Spin-orbit coupling in the solar system, Astronom. J. 71, n. 6 (1966), 425-438.

17. M. Guzzo, E. Lega and C. Froeschlé, On the numerical detection of the stability of chaotic motions in quasi-integrable systems, Physica D 163 (2002), 1-25.

18. J. Henrard, The adiabatic invariant in classical mechanics, Dynamics Reported 2, new series (1993), 117-235.

19. H. Hussmann and T. Spohn, Thermal orbital evolution of Io and Europa, Icarus 171 (2004), 391-410.

20. S.Y. Kim and D.S. Lee, Transition to chaos in a dissipative standardlike map, Phys. Rev. A 45, n. 8 (1992), 5480-5487.

21. J. Laskar, Frequency analysis for multi-dimensional systems. Global dynamics and diffusion, Physica D 67 (1993), 257-281. 
22. J. Laskar, C. Froeschlé and A. Celletti, The measure of chaos by the numerical analysis of the fundamental frequencies. Application to the standard mapping, Physica D 56 (1992), 253-269.

23. E. Lega and C. Froeschlé, Numerical investigations of the structure around an invariant KAM torus using the frequency map analysis, Physica D 95 (1996), 97-106.

24. G.J.F. MacDonald, Tidal friction, Rev. Geophys. 2 (1964), 467-541.

25. F. Marzari and S.J. Weidenschilling, Mean Motion Resonances, Gas Drag, and Supersonic Planetesimals in the Solar Nebula, Cel. Mech. dyn. Astr. 82, n. 3 (2002), 225-242.

26. S.J. Peale, The free precession and libration of Mercury, Icarus 178 (2005), 4-18.

27. H. Poincarè, Les Methodes Nouvelles de la Mechanique Celeste, Gauthier Villars, Paris, 1892.

28. C. L. Siegel, and J. K.Moser, Lectures on Celestial Mechanics, Springer-Verlag, Berlin, 1971.

29. G. Schmidt and B.W. Wang, Dissipative standard map, Phys. Rev. A 32, n. 5 (1985), 2994-2999.

30. V. Szebehely, Theory of orbits, Academic Press, New York and London, 1967.

31. S.J. Weidenschilling and A.A. Jackson, Orbital resonances and PoyntingRobertson drag, Icarus 104, n. 2 (1993), 244-254.

32. W. Wenzel, O. Biham and C. Jayaprakash, Periodic orbits in the dissipative standard map, Phys. Rev. A 43, n. 12 (1991), 6550-6557. 